\title{
Real-time observation of antigen-antibody association using a low-cost biosensing system based on photonic bandgap structures
}

\author{
J. G. Castelló, ${ }^{1, *}$ V. Toccafondo, ${ }^{1}$ J. Escorihuela, ${ }^{2}$ M. J. Bañuls, ${ }^{2}$ A. Maquieira, ${ }^{2}$ and J. García-Rupérez ${ }^{1}$ \\ ${ }^{1}$ Nanophotonics Technology Center, Universitat Politècnica de València, Camino de Vera s/n, 46022 Valencia, Spain \\ ${ }^{2}$ Centro de Reconocimiento Molecular y Desarrollo Tecnológico, Departamento de Química, \\ Universitat Politècnica de València, Camino de vera s/n, 46022 Valencia, Spain \\ *Corresponding author: jagarcas@ntc.upv.es
}

Received June 27, 2012; revised July 26, 2012; accepted July 26, 2012;

posted July 30, 2012 (Doc. ID 171333); published August 29, 2012

\begin{abstract}
In this letter, we present experimental results of antibody detection using a biosensor based on photonic bandgap structures, which are interrogated using a power-based readout technique. This interrogation method allows a realtime monitoring of the association process between the antigen probes and the target antibodies, allowing the instantaneous observation of any interaction event between molecules. because etunable lasers and optical spectrum analyzers are avoided for the readout, a drastic reduction of the final cost of the platform is obtained. Furthermore, the performance of the biosensing system is significantly enhanced due to the large number of data values obtained per second. () 2012 Optical Society of America

OCIS codes: $130.6010,130.5296$.
\end{abstract}

The interest in developing highly sensitive biosensors to identify and quantify a wide range of molecules has remarkably been increasing during the last decades. Thanks to their high potential, biological sensors can be applied in multiple fields such as medical diagnostics, food safety control, or environmental protection. Among the existing techniques to perform the detection, many require the use of labels (e.g., fluorofor, enzyme, particles) due to the lack of sensitivity to detect analytes at low concentrations. This fact leads to the necessity of developing much more sensitive biosensing devices able to perform the detection without physically modifying the sample through the use of labels.

Integrated optical devices based on silicon on insulator (SOI) are one of the main candidates to face this challenge [1]. Their extremely high sensitivity due to the high interaction of the evanescent field with the target analytes on the sensors' surface enables detection of low concentrations. The use of SOI-based structures also allows a drastic reduction of the sensors' footprint, which makes it possible to integrate many of them on a single chip to perform multianalyte detection. Moreover, the use of complementary metal-oxide semiconductor fabrication processes allows cost-effective mass production of these biosensors.

Among the most popular structures employed for the development of planar integrated photonic biosensors, we can find those based on microring resonators [2] or periodic structures [3,4]. Current interrogation methods for such biosensing devices are based on tracking the shift of any characteristic feature of their transmission spectrum, which must be continuously acquired. To this aim, it is necessary to use either a tuneable laser or an optical spectrum analyzer (OSA), which are significantly expensive. Moreover, they are bulky and heavy, making them not suitable for the development of compact and portable lab-on-a-chip (LOC) devices. In addition, this readout configuration may take several seconds to even minutes to acquire each sensor's spectrum. Different design variations of these structures have been reported by several groups in order to enhance the sensitivity, create slots [5,6], or introduce cavities [7], but not leading to a simplification of the system or a reduction of the cost in order to make it more practical for the implementation of photonic-based LOC devices. To tackle these issues, other configurations that do not require the use of tuneable elements have been proposed, such as those based on Mach-Zehnder interferometers [8] or more novel configurations such as Vernier-cascaded ring resonators [9] or bimodal waveguides [10]. However, the large footprint of these structures significantly limits the integration level of the final device. This issue can be overcome by folding the waveguides as in [11].

In a previous work [12], we presented a novel powerbased readout technique to perform the interrogation of photonic bandgap (PBG) - based sensing devices. The working principle of this technique is schematically illustrated in Fig. 1. Instead of directly measuring the

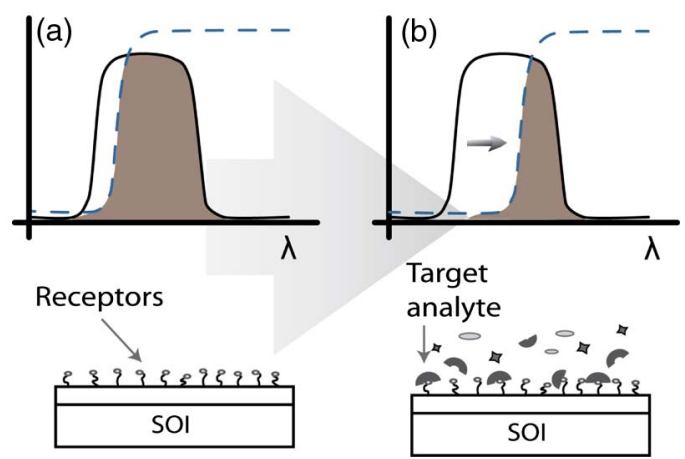

Fig. 1. (Color online) Schematic illustration of the working principle of the power-based biosensing technique where a filtered broadband source (solid curve) is aligned with the edge of the PBG (dashed curve). (a) Initial state, where only the bioreceptors are attached to the sensor's surface. (b) Induced shift of the PBG when the target analyte binds to the receptor. The shaded area represents the output power at each state given by the overlap between the source and the sensor's response. 
position of the PBG and tracking its shift, this wavelength shift is indirectly tracked by measuring the variation of the output power. This is achieved by exciting the PBGbased biosensor using a filtered broadband source (with a certain bandwidth), which is aligned with the edge of the PBG. The overlap between the source and the sensing structure determines the amount of output power. A change of the refractive index on the sensor's surface, which might be due to the binding of a target analyte, provokes a shift of the position of the PBG, leading to a variation of the output power, which is directly used to perform the sensing.

This new platform leads to several benefits, which are mainly derived from avoiding the use of a tuneable laser or an OSA for the readout. This fact allows significant reduction of the final cost of the platform, real-time observation because the output power is continuously monitored using a power meter, and a simpler analysis of the data because the sensing information is directly obtained from the output power without needing to perform any complex data processing to fit and track any spectral feature of the sensor's spectrum. Additionally, the use of simply a broadband source and a power meter for the readout also might allow their integration in the same chip as the biosensor, leading to a fully integrated device with a pure electro-optic access.

In this letter, we use both the proposed power-based and the typical spectral-based readout techniques to analyse the association process of the bovine serum albumin (BSA) - anti BSA antigen — antibody complex studied in microarrays [13], demonstrating a remarkable enhancement of the biosensor performance when the powerbased readout technique is used.

For the experiments, we have used the SOI onedimensional (1D) periodic structure shown in Fig. 2(a) as the photonic biosensor, which has been fabricated at Commissariat à l'énergie atomique et aux énergies alternatives-Laboratoire d'Électronique de Technologie de l'Information in the frame of the European ePIXfab platform. The dimensions of the $220 \mathrm{~nm}$ thick photonic structure are shown in Fig. 2(a). These parameters provide a PBG edge around $1550 \mathrm{~nm}$ as shown in Fig. 2(b). The fabricated structure consists of 101 periodic elements.

In order to deliver the target sample to the sensing structure, a $400 \mu \mathrm{m}$ wide and $50 \mu \mathrm{m}$ high SU8 microfluidic channel with two access reservoirs has been fabricated on top of the sensing structure by UV lithography. The
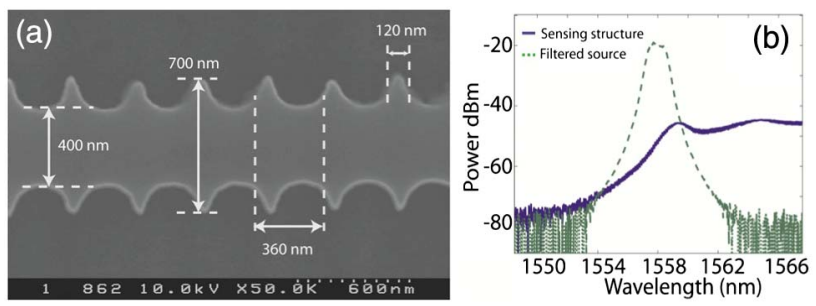

Fig. 2. (Color online) (a) Scanning electron microscope image of the 1D SOI periodic structure used as biosensor. Its dimensions are depicted. (b) Measured spectrum of the 1D SOI periodic structure (solid blue) and the filtered source (dashed green).

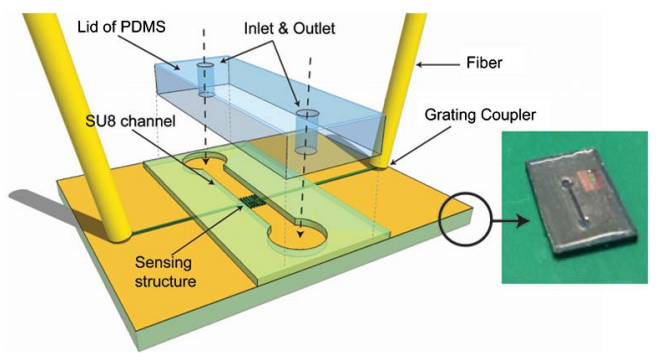

Fig. 3. (Color online) Schematic illustration of the sensing device with the top SU8 microfluidic channel closed using a PDMS lid. Grating couplers are used to couple the light to the photonic sensor. A picture of the chip used in the experiments is also shown.

microfluidic channel is closed using a polydimethylsiloxane (PDMS) lid with two access ports, as depicted in Fig. 3. Liquids are introduced into the microfluidic channel using Teflon tubes at $20 \mu \mathrm{l} / \mathrm{min}$.

As mentioned above, the spectral shift of the PBG of the sensing structure is induced by a change in the refractive index of the surrounding medium. However, for biosensing purposes, the PBG shift must be only induced by the target biomolecules to be detected. This selectivity is achieved by a proper biofunctionalization of the sensor's surface with specific capture probes.

Before the biofunctionalization of the sensor, the chip was immersed in piranha solution to remove any organic residue and increase the concentration of the $\mathrm{OH}$ groups to activate the surface. The functionalization was carried out in-line by flowing the chemicals over the sample. First, a 5 vol.\% solution of 3-(triethoxysilyl) propylisocyanate (ICPTS) in 2-propanol was flowed during $30 \mathrm{~min}$ for the silanization of the silicon surface. Immediately after this step, the antigen probes were immobilized by flowing a $10 \mu \mathrm{g} / \mathrm{ml}$ BSA solution in phosphate buffer saline (PBS) $1 \times$ during 50 min to covalently bind the antigen to the isocyanate end of the ICPTS. A rinse step with PBS $1 \times$ to remove any non covalently attached molecules was performed before blocking the remaining isocyanate moieties by flowing $0.1 \mathrm{M}$ ethanolamine in PBS $1 \times$.

Light from a super luminescent diode (Covega SLD1005S) broadband source with a flat response around $1550 \mathrm{~nm}$ was coupled to the photonic chip. Once the light passed through the sensing structure, the output signal from the chip was collected and divided using a power splitter. In one arm, the transmission spectrum of the PBG sensor was continuously monitored using an OSA (Ando AQ6317C) with $10 \mathrm{pm}$ resolution and $5 \mathrm{~nm}$ span around the PBG edge. In the other arm, the signal from the PBG sensor was filtered using a $0.8 \mathrm{~nm}$ bandwidth tuneable optical filter (DiconTF-1550-0.8) and continuously measured using a power meter (Newport 1931-C+918D-IR-OD1 photodetector). Note that this configuration, where the PBG sensor response is filtered at the output, is equivalent to filtering the excitation source at the input; this configuration is preferred this time because it allows the interrogation of the biosensor using simultaneously the typical spectral-based and the novel power-based readout techniques for their comparison.

Once the sensor was coated with the BSA antigen probes, two different concentrations of antiBSA antibody 

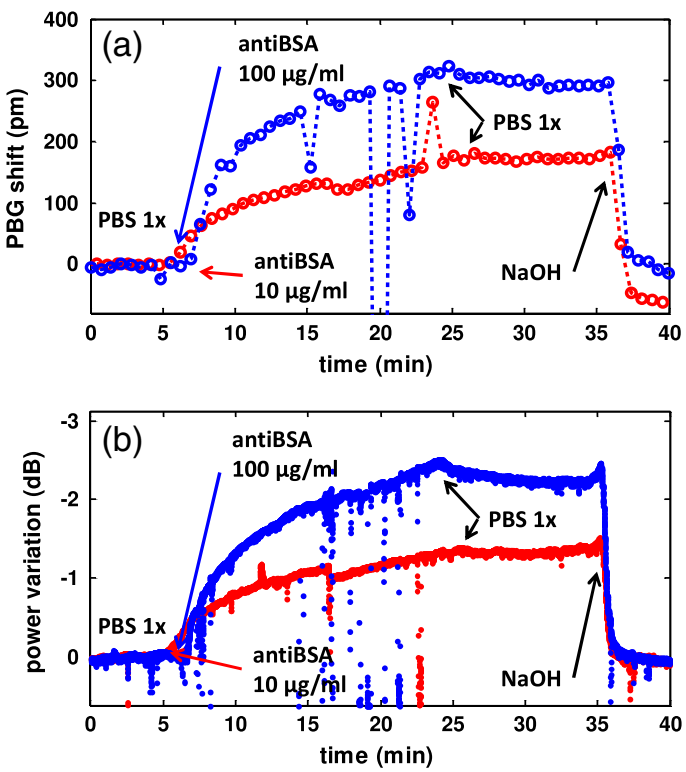

Fig. 4. (Color online) Time evolution of the PBG biosensor response using (a) direct spectral-based readout and (b) indirect power-based readout. For both readout methods, red and blue points depict the output signal for $10 \mu \mathrm{g} / \mathrm{ml}$ and $100 \mu \mathrm{g} / \mathrm{ml}$ of antiBSA, respectively. Arrows indicate the instant of each injection.

were flowed. Figure 4 shows the temporal evolution of the PBG biosensor response to the binding of the antiBSA to the BSA probes using both interrogation techniques. After each detection, a final regeneration step using $20 \mathrm{mM} \mathrm{NaOH}$ was carried out to remove bound antiBSA from the sensor's surface.

From curves in Fig. $\underline{4}$, we can confirm that the sensor response is the same using both readout techniques, because shifts in the PBG position are directly translated into output power variations. Therefore, as the sensing structure is the same in both cases, any variation in the final sensing performance is determined only by how the readout is made.

Using the power-based readout technique, the number of sensing data points is significantly higher than when using a spectral-based readout ( $\sim 10$ data points per $\mathrm{s}$ vs. 1 data point each $\sim 42 \mathrm{~s}$ ), which enables real-time measurements and the instantaneous observation of any event produced in the sensor's surface.

Real-time measurement also allows easy removal of any artefact caused by effects such as air microbubbles flowing over the sensor. For instance, we can see in Fig. 4(a) for the spectral-based readout how three negative glitches occur at temporal positions of 17,20 , and $22 \mathrm{~min}$, which are caused by air microbubbles. Because no other data point is acquired until $\sim 1$ min later, even though air bubbles have already passed away, sensing information is lost. On the other hand, the high temporal resolution of the power-based readout technique permits correction of this effect by simply removing those concrete power values from the sensing curve by data processing while preserving the whole information from the binding process, which returns to the correct position as soon as the undesired effect finishes.
Finally, the fact that many more data points are obtained when using the power-based readout technique allows reducing the noise by simply performing a temporal averaging, which is directly translated into a reduction of the detection limit without compromising the sensitivity of the biosensor device. For instance, using an averaging window of $30 \mathrm{~s}$, the noise level is reduced by a factor of $\sim 2$, and thus the detection limit.

In conclusion, we have shown how the performance of a PBG biosensor can be significantly increased by simply changing the readout approach. Using a novel powerbased readout technique, we are able to carry out a real-time monitoring of the sensor, acquiring more than 400 times more sensing data than typical spectral-based readout techniques. This allows the observation of any instantaneous event in the sensor's surface as well as reducing the influence of any artefact or undesired effect. Moreover, the detection limit can be significantly enhanced by simply performing a temporal averaging. Furthermore, this power-based readout technique avoids the use of tuneable equipment, which leads to a significant reduction of the cost, size, and weight of the final biosensing system, making it suitable for portable and low-cost LOC devices.

This work was partially funded by the European Commission under contract FP7-295043-BELERA, from the Spanish Ministerio de Ciencia e Innovación (MICINN) under contracts TEC2008-06333 and CTQ2010-15943 (subprogram BQU), and from Generalitat Valenciana through the PROMETEO grants 2010-008 and 2012-087.

\section{References}

1. M. S. Luchansky and R. C. Bailey, Anal. Chem. 84, 793 (2012).

2. A. J. Qavi and R. C. Bailey, Ang. Chem.-Int. Ed. 49, 4608 (2010).

3. J. García-Rupérez, V. Toccafondo, M. J. Bañuls, J. G. Castelló, A. Griol, S. Peransi-Llopis, and Á. Maquieira, Opt. Express 18, 24276 (2010).

4. V. Toccafondo, J. García-Rupérez, M. J. Bañuls, A. Griol, J. G. Castelló, S. Peransi-Llopis, and A. Maquieira, Opt. Lett. 35, 3673 (2010).

5. T. Claes, J. G. Molera, K. De Vos, E. Schacht, R. Baets, and P. Bienstman, IEEE Photon. J. 1, 197 (2009).

6. M. G. Scullion, A. Di Falco, and T. F. Krauss, Biosens. Bioelectron. 27, 101 (2011).

7. S. Zlatanovic, L. W. Mirkarimi, M. M. Sigalas, M. A. Bynum, E. Chow, K. M. Robotti, G. W. Burr, S. Esener, and A. Grot, Sens. Actuators B 141, 13 (2009).

8. B. Sepúlveda, J. S. Río, M. Moreno, F. J. Blanco, K. Mayora, C. Domínguez, and L. M. Lechuga, J. Opt. A 8, S561 (2006).

9. T. Claes, W. Bogaerts, and P. Bienstman, Opt. Lett. 36, 3320 (2011).

10. K. E. Zinoviev, A. B. González-Guerrero, C. Domínguez, and L. M. Lechuga, J. Lightwave Technol. 29, 1926 (2011).

11. A. Densmore, M. Vachon, D.-X. Xu, S. Janz, R. Ma, Y.-H. Li, G. Lopinski, A. Delâge, J. Lapointe, C. C. Luebbert, Q. Y. Liu, P. Cheben, and J. H. Schmid, Opt. Lett. 34, 3598 (2009).

12. J. G. Castelló, V. Toccafondo, P. Pérez-Millán, N. S. Losilla, J. L. Cruz, M. V. Andrés, and J. García-Rupérez, Opt. Lett. 36, 2707 (2011).

13. G. Krishnamoorthy, J. B. Beusink, and R. B. M. Schasfoort, Anal. Methods 2, 1020 (2010). 